\title{
Interference, Coupling, and Nonlinear Control of High-Order Modes in Single Asymmetric Nanoantennas.
}

\author{
Martina Abb, ${ }^{1}$ Yudong Wang, ${ }^{2}$ Pablo Albella, ${ }^{3}$ C. H. \\ de Groot, ${ }^{2}$ Javier Aizpurua, ${ }^{3}$ and Otto L. Muskens ${ }^{1}$ \\ ${ }^{1}$ SEPnet and Physics and Astronomy, \\ Faculty of Physical and Applied Sciences, \\ University of Southampton, Highfield, \\ Southampton SO17 1BJ, United Kingdom \\ ${ }^{2}$ Electronics and Computer Science, \\ Faculty of Physical and Applied Sciences, \\ University of Southampton, Highfield, \\ Southampton SO17 1BJ, United Kingdom \\ ${ }^{3}$ Donostia International Physics Center, DIPC, \\ and Centro de Fisica de Materiales CSIC-UPV/EHU, \\ Paseo Manuel Lardizabal 4, Donostia-San Sebastian 20018, Spain
}

(Dated: June 18, 2012)

\begin{abstract}
We investigate theoretically and experimentally the structure of plasmonic modes in individual asymmetric dimer antennas. Plasmonic near-field coupling of high-order modes results in hybridization of bright and dark modes of the individual nanorods leading to an anticrossing of the coupled resonances. For two bright modes, hybridization results in a capacitive redshift and superradiant broadening. We show that the properties of asymmetric dimers can be used for nonlinear control of spectral modes and demonstrate such a nonlinear effect by measuring the modulation of a hybrid asymmetric dimer - ITO antenna. With use of full electrodynamical calculations we find that the properties of the near-field nonlinear responses are distinctly different from the far field, which opens up new routes for nonlinear control of plasmonic nanosystems.
\end{abstract}

Keywords: plasmon; nanoantenna; asymmetric dimer; Fano resonance; nonlinear response; figure of merit 
In recent years there have been tremendous advances in the design and fabrication of new types of nanoscale optical elements displaying surface plasmon resonances. Plasmonic devices have been very successful in a wide range of applications, including surface-enhanced Raman $^{1,2}$ and biosensing for healthcare, near-field optical spectroscopy, ${ }^{3,4}$ enhancement of light emission ${ }^{5,6}$ and photodetectors, ${ }^{7,8}$ and plasmonic metamaterials. ${ }^{9,10}$ Analogous to radiowave antennas, plasmonic nanoantennas are designed to efficiently convert optical radiation into a nanometer-sized volume. ${ }^{11}$ Plasmonics holds promise as a new paradigm for achieving truly nanoscale, ultrafast optical devices in integrated photonic circuits. ${ }^{12-14}$ Active control of the properties of electromagnetic resonances in nanoantennas is generally considered as an important landmark toward the development of transistor-type nanodevices capable of manipulating the flow and emission of light.

In addition to standard dipole gap antennas, many new antenna designs have been developed in recent years to optimize specific properties such as directivity and bandwidth, e.g. bowtie antennas, multi-particle clusters supporting Fano-type resonances ${ }^{15,16}$ or 3D, layered antenna designs. ${ }^{17}$ These more complex designs also offer new routes for nonlinear control beyond the standard symmetric dimer structures because of the appearance of narrower spectral features associated with nonradiative plasmon modes or, generally, a more intricate dependence of the mode spectrum on the nanoscale geometry. ${ }^{18}$

Recently a simple new model system has been proposed theoretically based on the coupling between two nanorods of different length. ${ }^{19-21}$ Some effects of symmetry breaking have been investigated experimentally in colloidal nanoparticle heterodimers consisting of different sized nanorods and nanospheres. ${ }^{22-24}$ As an extension of the highly successful dimer gap antenna concept, asymmetric dimers may be of interest for a variety of applications, such as sum-frequency generation, multi-frequency sensors, and Raman scattering. ${ }^{25-27}$ Here, we present a combined experimental and theoretical study to address the optical response of individual asymmetric dimers both in the linear and nonlinear regime. Comparison of experimental and calculated spectra confirm the interference and coupling of higher-order plasmonic modes in the nanorods, ${ }^{28-32}$ providing evidence of the existence of two types of coupling depending on the radiative nature of the modes. 


\section{Results and Discussion}

Experimental spectra of single antennas. Figure 1 (center) shows Scanning Electron Microscopy images of selected asymmetric dimer antennas with a gap of $20 \mathrm{~nm}$. The antennas consist of a short $\left(L_{1}\right)$ and a long $\left(L_{2}\right)$ nanorod arm. The length of the long nanorod $\operatorname{arm}\left(L_{2}\right)$ is varied from $360 \mathrm{~nm}$ to $550 \mathrm{~nm}$ in steps of $10 \mathrm{~nm}$. Extinction spectra of the individual asymmetric dimer antennas are shown in panel b) of Fig. 1, with corresponding calculations shown in panel a). The spectra show a pronounced dependence on the degree of asymmetry, with good agreement between simulations and experiment. Dashed lines are guides to the eye and the different labels from A to E mark points of reference in the spectra for easy comparison with calculations presented further below (Fig. 2). We can identify the spectral peaks $\mathrm{B}$ and $\mathrm{E}$ as corresponding to the fundamental dipole mode of the short rod $\left(L_{1}=\lambda_{\text {eff }} / 2\right.$ with $\lambda_{\text {eff }}$ the effective resonance wavelength $\left.{ }^{28}\right)$. As $L_{1}$ decreases, $L_{2}$ increases in length producing a redshift of the resonance from $B$ to E. At a length of $L_{2} \approx 420 \mathrm{~nm}$, the dipole mode splits into two branches (marked by points $\mathrm{C}$ and $\mathrm{D}$ in the graph). For $L_{2}=460 \mathrm{~nm}$, another crossing of modes is observed without splitting. The fundamental dipole mode for the long antenna $\left(L_{2}=\lambda_{\text {eff }} / 2\right)$ is located at a longer wavelength, i.e. outside of our experimental window. However, we can identify the spectral peak at point A as corresponding to a higher-order bright mode of the long antenna $\left(L_{2}=3 \lambda_{\text {eff }} / 2\right)$.

Figure 1 confirms experimentally that the crossing of higher-order modes of the individual nanorods results in a hybridization of the asymmetric antenna modes. To get a complete picture of the underlying interference and coupling between modes, we numerically investigate the interplay between different multipolar modes as a function of the ratio of the lengths of the two antenna segments $\left(L_{1} / L_{2}\right)$ using model calculations of the optical response of an asymmetric antenna in vacuum. In our calculations the antenna dimers are modelled as two cylindrical rods with radius $R=15 \mathrm{~nm}$ and hemispherical endcaps. The total length, including endcaps but excluding the gap, was set to $600 \mathrm{~nm}$ as in the experiments. We also calculated the additional case where the antennas show a total rod length of $L_{t o t}=1000 \mathrm{~nm}$, since this situation reveals the interferences of even higher modes (up to $L_{1,2}=5 \lambda_{e f f} / 2$ ).

Modelling of mode interactions in asymmetric dimers. As a first step towards the modeling of the mode interactions in the asymmetric dimer, we first study the modes of each of the segment rods individually. For an overview of the modes sustained by the individual 
nanorods, we consider the effective wavelength scaling formula given by Novotny, ${ }^{33}$ which in the approximation $L>>R$ can be expressed as:

$$
\begin{aligned}
\lambda \approx & \left(\frac{\lambda_{p} \epsilon_{s}}{0.12 \sqrt{\epsilon_{\infty}+141.04 \epsilon_{s}}}\right) \cdot\left(\frac{n L}{4 \pi R}\right. \\
& \left.-13.74-\frac{0.12}{\epsilon_{s}}\left[\sqrt{\epsilon_{\infty}+141.04 \epsilon_{s}}\right]-\frac{2}{\pi}\right) .
\end{aligned}
$$

Here $\lambda_{p} \approx 138 \mathrm{~nm}$ is the plasma wavelength of gold, $\epsilon_{\infty} \approx 11$ the infinite frequency limit of the dielectric function for gold, $\epsilon_{s}$ the surrounding medium, $n$ is the order of the mode and $L$ the length of the nanorod including the endcaps.

Figure 2a) shows the calculated positions for the modes of the individual rods $L=L_{1}$ (short) and $L=L_{2}$ (long) constituting the asymmetric dimer for the case where the total length $L_{t o t}=600 \mathrm{~nm}$, calculated according to Eq. (1). We plot in solid lines the radiative modes and in dashed lines the nonradiative modes. Modes are radiative if they have a nonzero net dipole moment; the dipole moment depends on the order of the mode, i.e. odd multiples of $\lambda_{\text {eff }} / 2$ are radiative and even multiples are nonradiative. ${ }^{28}$ The modes corresponding to the rod on the left blueshift as the rod length $L_{1}$ is decreased, while those of the rod on the right redshift because of the increase in length $L_{2}$. This situation leads to the coexistence of modes that belong to each of the segments of the antenna occurring at the same wavelength, and thus causing interference. Several crossings between the fundamental mode of $L_{1}$ and higher order modes of $L_{2}$ are indicated by circles in Fig. 2a).

Figure 2a) represents the presence of modes in the system, but the actual excitation of each mode is determined by how effectively the external driving field couples to the particular mode. Because of the symmetry, dark modes as the ones represented by the dashed lines in Fig. 2a,e) are not observed in simulations or in experiments since they cannot be excited using standard plane wave incidence configurations. Also the simple model of Eq. (1) does not take into account the effect of coupling on the position of the modes, but gives a good estimate of the wavelengths where the coupling of modes occurs. The complete effect of the interaction can be unraveled with use of full electrodynamical calculations. Figures 2b-d) show the results for antennas formed by two asymmetric segments of two different values of the length of the gap between the rods, $100 \mathrm{~nm}$ (b), $20 \mathrm{~nm}$ (c) and $2 \mathrm{~nm}$ (d). For the $100 \mathrm{~nm}$ gap, where the interaction is small, the calculations correspond quite well with the expected positions of the individual modes using the simple model. As expected, the dark modes 
(dashed lines) are not observed in the far-field extinction. Only a small dip is observed around the first crossing point in Figure 2b), which is the first onset of a hybridization between dark and bright modes. This hybridization is observed more clearly in Fig. 2d) for the case of the dimer with a $20 \mathrm{~nm}$ gap. In such a case, the hybridization is pronounced with splitting of modes at length ratios of about $L_{1} / L_{2} \approx 1: 2$ and $1: 4$. This behavior is observed into greater detail for the nanoantenna with a total length of $L_{\text {tot }}=1000 \mathrm{~nm}$ as shown in Fig. 2(e-h). For the 2-nm gap, the splitting exceeds the linewidth of the resonances, indicating that the two nanorods are in the strong coupling regime.

To investigate into more detail the induced splitting, we plot both field enhancement and phase of the local near fields in Fig. 3 for modes C and D in panel c). Modes C and D are spectrally located at each branch of the mode splitting corresponding to a dimer of total length $L_{t o t}=600 \mathrm{~nm}$ and long arm length $L_{2}=420 \mathrm{~nm}$. The near-field maps clearly show the bonding nature of the mode at $950 \mathrm{~nm}$ (mode D), while the mode at $870 \mathrm{~nm}$ (mode C) shows an anti-bonding profile with zero intensity in the gap. ${ }^{34-36}$ Another feature visible in the phases are the induced charges at the tip of the longer rod caused by the near-field coupling to the bright mode of the short rod.

Strength of coupling. The strength of the coupling between modes can be tuned by the distance between the two antenna segments, as can be observed in Fig 4a) where we plot the extinction spectrum of the antenna versus gap size for an asymmetric antenna. This behavior is confirmed experimentally in Fig. 4b) where the splitting of modes is measured in the differential reflectance of asymmetric antennas with different gap sizes of 10, 30 and $40 \mathrm{~nm}$. This splitting corresponds to the avoided crossing of dark and bright modes due to coupling and gives rise to electromagnetically-induced transparency (EIT). ${ }^{37,38}$ EIT is an effect driven by the interference between excitation pathways that can occur both in quantum and classical oscillator systems. Coupling between a bright and a dark mode results in a near-perfect transparency of the system on an otherwise strong absorption line. The phenomenon is based on the modification of the radiative mode by the presence of a dark, nonradiative mode, which is excited through the near-field coupling with the radiative dipolar mode.

In Fig. 4a), we can observe how strongly the EIT splitting is dependent on the dimer separation. In our system, the splitting first occurs at a separation of $50 \mathrm{~nm}$. With decreasing separation distances, we see that the splitting becomes asymmetric. The bonding mode 
redshifts in cubic dependence of the gap size, as expected for dipole-dipole interaction. The antibonding mode, however, blueshifts more slowly. This is caused by the interaction of the plasmon in the shorter nanorod with plasmons of much higher order in the other rod, as already found in the case of spherical nanoparticle dimers. ${ }^{34}$ The suppression of the bright mode as a consequence of the coupling with the dark mode is illustrated in Fig. 4c) where the near-field distribution around the antenna is represented for decreasing gap sizes from $200 \mathrm{~nm}$ down to $2 \mathrm{~nm}$ and at a wavelength of $910 \mathrm{~nm}$, i.e. in the center of the EIT window. For the large antenna gap, the short nanorod shows a strong dipole mode; however, this mode is almost completely suppressed for a small antenna gap of $2 \mathrm{~nm}$ through coupling with the long rod. We estimate the amount of energy transfer from the short rod to the long rod by integrating the square of the near field magnitude $|E|$ in Fig. 4c) over a contour around each individual rod at a distance of $2 \mathrm{~nm}$ away from the edge of the rod. Figure $4 \mathrm{~d}$ ) shows the resulting values for the integrated $|E|^{2}$ for the two rods, normalized to the value for the the short rod in absence of the long rod. At large gap sizes only a small fraction of the mode energy is transferred to the long rod via far-field coupling. The integrated $|E|^{2}$ of the long rod reaches a maximum value for a gap size around $30 \mathrm{~nm}$. This maximum arises because of the combined effect of an increased energy transfer and a suppression of the total mode intensity for small gap sizes, as is illustrated by the black triangles in Fig. 4d). In order to assess the relative efficiency of energy transfer from the short rod to the long rod, we also plot in Fig. 4e) the integrated $|E|^{2}$ for the two rods normalized to their sum value. This method divides out the variation of the total mode intensity, and thus gives a measure of the efficiency of energy transfer to the long rod. We find a monotonous increase toward shorter gap sizes in Fig. 4e), which levels off for gaps below $10 \mathrm{~nm}$. A maximum transfer efficiency of $76 \%$ is found at a gap size of $10 \mathrm{~nm}$. The dependence on wavelength of the integrated $|E|^{2}$ values, for a fixed gap size of $10 \mathrm{~nm}$, is shown in Fig. 4(f,g). Indeed the maximum of energy transfer efficiency to the long rod occurs at $910 \mathrm{~nm}$ wavelength, i.e. the center of the EIT window of Fig.4a).

On the other hand, crossing of two bright modes does not result in a splitting, as in EIT. Instead, the coherent superposition of two bright modes results in a capacitive redshift and a superradiant broadening as can be most clearly observed in the spectra of Fig. 1 for $L_{2}=480 \mathrm{~nm}$. In addition, we find by comparing the efficient coupling case in Fig. 2c,d) with the weakly coupled case in Fig. 2b), that the strength of bright modes is significantly 
affected even away from the crossings. For example, the $3 \lambda_{\text {eff }} / 2$ mode of the long rod (mode A) is significantly suppressed below and enhanced above the crossing with the fundamental $\lambda_{\text {eff }} / 2$ mode of the short rod (mode B). This suppression as a function of coupling strength is also observed in Fig $4 \mathrm{a}$ ) at the wavelength of $0.7 \mu \mathrm{m}$ and shows that the short rod couples into the modes of the long rod and vice versa, even for configurations where their individual resonances are not spectrally overlapping.

Nonlinear response of asymmetric dimers. We now consider the effect of mode coupling and EIT on the nonlinear response of the asymmetric dimers. We explore the response of an antenna-ITO hybrid system as a way to achieve a large optical nonlinearity of the combined system, ${ }^{39}$ as previously reported for symmetric antennas. The enhancement of the nonlinear response of antenna-ITO hybrids is based on picosecond energy transfer of electronic and thermal excitations generated in the gold antenna by a short pulsed laser excitation. Fast electrons are excited in the gold nanoantenna and injected from the antenna into the ITO. The following thermalization of these electrons leads to heating of the immediate surroundings and subsequent migration of electrons from the heated region. This interaction produces a nonlinear refractive index change of the ITO substrate directly surrounding the antenna, enhancing the hybrid antenna-ITO nonlinear response.

We experimentally investigated the nonlinear response of an asymmetric dimer antenna with long rod length $L_{2}=420 \mathrm{~nm}$. The differential reflectance corresponding to extinction obtained through the spatial modulation method is shown in Fig. 5a), showing the EITcrossing of the modes $\mathrm{C}$ and $\mathrm{D}$ from Fig. 1. The fast, picosecond component of the nonlinear modulation signal, i.e. after subtraction of a thermal background, is shown in Fig. 5b) for a delay of 5 ps between the arrival of pump and probe pulses. The fast transient modulation has a typical decay time of 200 ps given by the cooling dynamics of the substrate. ${ }^{40}$ We can observe this time evolution of the nonlinear response at different wavelengths in Fig. 5e). In symmetric antennas, a redshift of the dipolar resonance was previously found resulting from a reduction in the free carrier density of the ITO substrate. ${ }^{39}$ In our case, the response of the asymmetric dimer shows at least three positive and two negative contributions as marked by the Greek symbols $\alpha$ to $\epsilon$. Comparison with the numerical model in Fig. 5c,d) confirms this trend around the EIT anti-crossing, showing a redshift for each arm of the splitting. For this calculation we used an initial free carrier concentration of $N_{0}=7.3 \times 10^{20} \mathrm{~cm}^{-3}$ in the ITO and an induced change in carrier concentration of $\Delta N=-6 \times 10^{19} \mathrm{~cm}^{-3}$ consistent 
with our earlier findings. ${ }^{39}$ The BEM-calculation of the differential extinction cross-section is shown in Fig. 5f) and exhibits the same structure of four contributions to the spectrum for the exact situation where the splitting occurs $\left(L_{2}=420 \mathrm{~nm}\right)$. This effect can be associated with the redshift of both the upper and lower branches of the hybridized modes. For these BEM calculations, we used a geometry where ITO was present only in the antenna gap, however the exact distribution of material around the antenna does not strongly influence the behavior for small changes for in the free-carrier concentration $\Delta N{ }^{39}$

Nonlinearity in the near-field. While the far-field shows a nonlinear response characterized by an overall redshift of the resonance spectrum, a more complex behavior is found for the near-field. In Fig. 6, we plot the calculated near-field distribution in the proximity of asymmetric antennas at different positions: at $5 \mathrm{~nm}$ to the left of the short rod tip (A1), at the midgap (A2) and at $5 \mathrm{~nm}$ to the right of the long rod tip (A3). The maps of A1 and A3 are governed by the modes of the short and long rods, respectively, away from the crossing points. It can be observed in these maps of the local field that, for the lengths where EIT is produced due to the crossing of bright and dark modes, the electromagnetic energy is transferred from the tip of the short rod (A1) to the long rod (A3) (see the bright spots in Fig. 6c), coinciding with the dark spots in Fig. 6a)). This effect can be of interest in many applications. It would be possible for example to position a molecule at the end point of the long antenna (A3) which will only be excited through coupling of the long rod with the bright mode of the short rod at the EIT condition. As was shown in Fig. 4e), the efficiency of energy transfer can reach $76 \%$; however the maximum near-field enhancement achieved in A3 is less than that of the short-arm dipole in A1, most prominently because of the difference in antenna length and the concomitantly larger mode volume.

The nonlinear response of the local field intensity, $\Delta I$, is shown in Fig. 6d)-f) for positions A1 - A3. Here, $\Delta I$ is defined as the difference of the intensity maps calculated using the modified free carrier density $N=7.3 \times 10^{20} \mathrm{~cm}^{-3}$ compared with those at the original carrier density $N_{0}$. The response above $1.2 \mu \mathrm{m}$ is associated with the dipole mode of the long antenna outside our spectral window and will not be discussed. Signatures of the bipolar response as found in the far-field cross-section are observed at all three positions. Position A3 however shows additional effects which cannot be explained by an overall nonlinear shift of the resonance wavelength, but which indicate the presence of other contributions in the nonlinear response. Particularly around the branches $\mathrm{C}$ and $\mathrm{D}$ only positive values 
are found for $\Delta I$. This positive nonlinear response is the strongest for the bonding mode (D). We attribute this effect to an increased coupling strength induced by the reduction of the refractive index in the antenna gap. It was observed in Fig. 5a) that the bonding mode depends more strongly on the coupling strength than the anti-bonding mode, which is consistent with the difference in $\Delta I$ for points $\mathrm{C}$ and $\mathrm{D}$ in Fig. 6f). Such a mechanism is of interest as it shows a distinct difference in nonlinear response associated with the EIT mode, which may be further optimized for applications.

Figure of Merit. Various structures supporting EIT and Fano-type resonances have been proposed and used in the recent literature. In the dolmen-type, pi, or heptamertype structures ${ }^{16}$ the combination of dipoles and/or multipoles oscillating in- and outof-phase results in a cancellation of fields and the appearance of a dark mode. In the asymmetric dimer, the coupling of a true dark mode with another dipole gives rise to the EIT transparency in analogy with molecular systems. ${ }^{38}$ Applications in nonlinear control are similar to those in sensing and benefit from a large sensitivity of the spectral mode structure on local changes in configuration and refractive index combined with enhancement of optical near-fields. The Figure of Merit (FOM) for such applications is defined by the ratio of the plasmon lineshift per refractive index unit, normalized to the spectral width, and allows a comparison of the performance of different types of structures. ${ }^{41}$ For small spectral shifts, the FOM is approximately proportional to the differential pump-probe cross-section $\Delta \sigma / \sigma$ in the wing of the resonance (assuming a single resonance without background). This equivalence can be seen from the fact that $\Delta \sigma / \sigma=0.5$ for a resonance shift equal to half the line width. At the maximum marked by $\gamma$ in Fig. 5 d) we find a value of $\Delta \sigma / \sigma=0.2$. The corresponding refractive index change of the ITO matrix used in this calculation of $\Delta n=0.056+0.009 i$ at the wavelength of $910 \mathrm{~nm}$ as shown in Fig. 7. This value results in a FOM of 3.6 at the $\gamma$ maximum. This FOM-value is comparable to that of other EIT-structures reported in literature. ${ }^{41}$ It should be noted that according to Fig. 4, the width of the EIT-dip, and therefore the FOM, depends on the gap size. A larger gap results in a smaller splitting and thus a higher Figure of Merit. 


\section{Conclusions}

In conclusion, we have investigated both experimentally and theoretically the interference and coupling of electromagnetic modes in asymmetric dimers. Asymmetric dimers are a relatively simple model system for studying effects of electromagnetic induced transparency. Their simplicity of design is of interest as the geometry in principle could be achieved using a variety of techniques including both top-down lithography as well as bottom-up fabrication methods such as colloidal chemistry or electrochemical templated growth. This structure thus opens a path for generation of simple structures that allow for manipulation of EIT and control of spectral resonances. In addition to the linear optical response of the asymmetric dimers, we have demonstrated the signature of EIT-splitting also in the nonlinear regime through picosecond modulation of the refractive index of the ITO substrate. The high sensitivity of coupled asymmetric nanorod dimers to the dielectric environment is of potential interest for nonlinear devices and plasmon resonance sensors.

\section{Methods}

Modelling. In order to reproduce the experimental situation as accurate as possible, the substrate and the fine details of the rectangular antenna geometry were considered in the full electrodynamical calculations of the optical response.A Finite-Difference in Time Domain (FDTD) method was used (Lumerical) to obtain the results for the antennas deposited on the substrate whereas systematic calculations of the dependence of the coupled resonances on the different antenna parameters were performed for cylindrical metallic rods in the absence of substrate with use of the Boundary Element Method (BEM). ${ }^{42}$

Materials. In our experiments, asymmetric dimer antennas of $25 \mathrm{~nm}$ thickness were fabricated on an ITO-coated glass substrate using e-beam lithography (JEOL JBX-9300FS) at $100 \mathrm{kV}$ and a dose of $650 \mu \mathrm{C} / \mathrm{cm}^{2}$. The width of all the antennas was kept fixed at $70 \mathrm{~nm}$.

Single-particle spectroscopy. Spectra of individual antennas were measured using a pulsed supercontinuum light source (Fianium, $40 \mathrm{MHz}, 4 \mathrm{ps}$ ) and a spatial modulation microscopy setup following the method introduced in Ref. ${ }^{39}$. The spatial modulation method is based on the detection of a small variation in optical reflection (or transmission) created by periodic displacement of the antenna in the focus of a microscope objective (Mitutoyo 
$100 \times$ NIR, $0.5 \mathrm{NA})^{43,44}$. The modulation signal was detected using a lock-in amplifier and was directly proportional to the extinction cross section of the antenna. To obtain extinction spectra, the optical wavelength was scanned using a double-prism subtractive mode monochromator. For nonlinear pump-probe experiments, we used the same spectroscopy setup in combination with a synchronized Ytterbium fibre amplifier, which was frequency doubled to a wavelength of $530 \mathrm{~nm}$.

Conflict of interest: The authors declare no competing financial interest.

Acknowledgement. P. Albella acknowledges the support received from the European Science Foundation for the activity entitled: "New approaches to Biochemical sensing with Plasmonic Nanobiophotonics" (PLASMON-BIONANOSENSE). J. A. acknowledges funding from the ETORTEK project inanoGUNE from the Basque Government and project FIS200766711-C01-01 of the Spanish Ministry of Innovation and Science. O. L. M. acknowledges support from EPSRC through Grant EP/J010758/1.

\section{References}

1 Rodriguez-Lorenzo, L.; Alvarez-Puebla, R. A.; Pastoriza-Santos, I.; Mazzucco, S.; Stephan, O.; Kociak, M.; Liz-Marzan, L. M.; Garcia de Abajo, F. J. Zeptomol Detection Through Controlled Ultrasensitive Surface-Enhanced Raman Scattering. J. Am. Chem. Soc. 2009, 131, 4616-4618.

${ }^{2}$ Kneipp, J.; Li, X.; Sherwood, M.; Panne, U.; Kneipp, H.; Stockman, M. I.; Kneipp, K. Gold Nanolenses Generated by Laser Ablation-Efficient Enhancing Structure for Surface Enhanced Raman Scattering Analysis and Sensing. Anal. Chem. 2008, 80, 4247-4251.

3 Schnell, M.; Garcia-Etxarri, A.; Huber, A. J.; Crozier, K.; Aizpurua, J.; Hillenbrand, R. Controlling the Near-Field Oscillations of Loaded Plasmonic Nanoantennas. Nat. Phot. 2009, 3, 287-291.

4 Schnell, M.; Garcia-Etxarri, A.; Alkorta, J.; Aizpurua, J.; Hillenbrand, R. Phase-Resolved Mapping of the Near-Field Vector and Polarization State in Nanoscale Antenna Gaps. Nano Letters 2010, 10, 3524-3528.

5 Giannini, V.; Fernandez-Dominguez, A. I.; Heck, S. C.; Maier S. A., Plasmonic Nanoantennas: 
Fundamentals and Their Use in Controlling the Radiative Properties of Nanoemitters. Chem. Rev. 2011, 111, 3888-3912.

${ }^{6}$ Curto, A. G.; Volpe, G.; Taminiau, T. H.; Kreuzer, M. P.; Quidant, R.; Van Hulst, N. F., Unidirectional Emission of a Quantum Dot Coupled to a Nanoantenna. Science 2010, 329, 930-933.

7 Knight, M. W.; Sobhani, H.; Nordlander, P.; Halas, N. J. Photodetection with Active Optical Antennas. Science 2011, 332, 702-704.

8 Tang, L.; Kocabas, S. E.; Latif, S.; Okyay, A. K.; Ly-Gagnon, D.-S.; Saraswat, K. C.; Miller, D. A. B., Nanometre-Scale Germanium Photodetector Enhanced by a Near-Infrared Dipole Antenna. Nat. Photon. 2008, 2, 226-229.

9 Valentine, J; Zhang, S.; Zentgraf, T.; Ulin-Avila, E.; Genov, D. A.; Bartal, G.; Zhang X., Three-Dimensional Optical Metamaterial with a Negative Refractive Index. Nature 2008, 455, 376-379.

10 Wurtz, G. A.; Pollard, R.; Hendren, W.; Wiederrecht, G. P.; Gosztola, D. J.; Podolskiy, V. A.; Zayats, A. V. Designed ultrafast optical nonlinearity in a plasmonic nanorod metamaterial enhanced by nonlocality. Nat. Nanotech. 2011, 6, 107-111.

11 Novotny, L.; van Hulst, N. Antennas for Light. Nat. Photon. 2011, 5, 83-90.

12 Brongersma, M. L.; Shalaev, V. The Case for Plasmonics. Science 2010, 328, 440-441.

13 MacDonald, K. F.; Samson, Z. L.; Stockman, M. I.; Zheludev, N. I. Ultrafast Active Plasmonics. Nat. Photon. 2009, 3, 55-58.

14 Large, N.; Abb, M.; Aizpurua, J.; Muskens, O. L. Photoconductively Loaded Plasmonic Nanoantenna as Building Block for Ultracompact Optical Switches. Nano Lett. 2010, 10, 1741-1746.

15 Verellen, N.; Sonnefraud, Y.; Sobhani, H.; Hao, F.; Moshchalkov, V. V.; Van Dorpe, P.; Nordlander, P.; Maier, S. A. Fano Resonances in Individual Coherent Plasmonic Nanocavities. Nano Lett. 2009, 9, 1663-1667.

16 Luk'yanchuk, B.; Zheludev, N. I.; Maier, S. A.; Halas, N. J.; Nordlander, P.; Giessen, H.; Chong, C. T. The Fano Resonance in Plasmonic Nanostructures and Metamaterials. Nat. Mater. 2009, 9, 707-715.

17 Liu, N.; Hentschel, M.; Weiss, T.; Alivisatos, A. P.; Giessen, H. Three-Dimensional Plasmon Rulers. Science 2011, 332, 1407-1410.

18 Alonso-Gonzalez, P.; Schnell, M.; Sarriugarte, P.; Sobhani, H.; Wu, C.; Arju, N.; Khanikaev, A.; 
Casanova, F.; Hueso, L. E.; Nordlander, P. et al. R. Real-Space Mapping of Fano Interference in Plasmonic Metamolecules. Nano Lett. 2011, 11, 3922-3926.

19 Yang, Z.-J.; Zhang, Z.-S.; Zhang, L.-H.; Li, Q.-Q.; Hao, Z.-H.; Wang, Q-Q. Fano Resonances in Dipole-Quadrupole Plasmon Coupling Nanorod Dimers. Opt. Lett. 2011, 36, 1542-1544.

20 Woo, K. C.; Shao, L.; Chen, H.; Liang, Y.; Wang, J.; Lin, H.-Q. Universal Scaling and Fano Resonance in the Plasmon Coupling between Gold Nanorods. ACS Nano 2011, 5, 59765986.

21 Huang, C.-P.; Yin, X.-G.; Kong, L.-B.; Zhu, Y.-Y. Interactions of Nanorod Particles in the Strong Coupling Regime. J. Phys. Chem. C 2010, 114, 21123-21131.

22 Brown, L. V.; Sobhani, H.; Lassiter, J. B.; Nordlander, P.; Halas, N. J. Heterodimers: Plasmonic Properties of Mismatched Nanoparticle Pairs. ACS Nano 2010, 4, 819832.

23 Slaughter, L. S.; Wu, Y.; Willingham, B. A.; Nordlander, P.; Link S. Effects of Symmetry Breaking and Conductive Contact on the Plasmon Coupling in Gold Nanorod Dimers. ACS Nano 2010, 4, 46574666.

24 Shao, L.; Fang, C.; Chen, H.; Man, Y. C.; Wang, J.; Lin, H.-Q. Distinct Plasmonic Manifestation on Gold Nanorods Induced by the Spatial Perturbation of Small Gold Nanospheres. Nano Lett. 2012, 12, 14241430 .

25 Danckwerts, M.; Novotny, L. Optical Frequency Mixing at Coupled Gold Nanoparticles. Phys. Rev. Lett. 2007, 98, 026104.

26 Petschulat, J.; Cialla, D.; Janunts, N.; Rockstuhl, C.; Hübner, U.; Möller, R.; Schneidewind, H.; Mattheis, R.; Popp, J.; Tünnermann, A. et al. Doubly Resonant Optical Nanoantenna Arrays for Polarization Resolved Measurements of Surface-Enhanced Raman Scattering. Opt. Expr. 2010, 18, 4184-4197.

27 Harutyunyan, H.; Volpe, G.; Quidant, R.; Novotny, L. Enhancing the Nonlinear Optical Response Using Multifrequency Gold-Nanowire Antennas. Phys. Rev. Lett. 2012, 108, 217403.

28 Aizpurua, J.; Bryant, G. W.; Richter, L. J.; García de Abajo; F. J., Kelley, B. K.; Mallouk, T. Optical Properties of Coupled Metallic Nanorods for Field-Enhancement Spectroscopy. Phys. Rev. B 2005, 71, 235420..

29 Bryant, G. W.; García de Abajo, F. J.; Aizpurua, J. Mapping the Plasmon Resonances of Metallic Nanoantennas. Nano Lett. 2008, 8, 631-636.

30 Payne, E. K.; Shuford, K. L.; Park, S.; Schatz, G. C.; Mirkin, C. A. Multipole Plasmon Resonances in Gold Nanorods. J. Phys. Chem. B 2006, 110, 2150-2154. 
31 Wei, H.; Reyes-Coronado, A.; Nordlander, P.; Aizpurua, J.; Xu, H. Multipolar Plasmon Resonances in Individual Ag Nanorice. ACS Nano 2010, 4, 2649-2654.

32 Schmidt, M. K.; Mackowski, S.; Aizpurua, J. Control of Single Emitter Radiation by Polarization- and Position-Dependent Activation of Dark Antenna Modes. Opt. Lett. 2012, 37, 1017-1019.

33 Novotny, L. Effective Wavelength Scaling for Optical Antennas. Phys. Rev. Lett. 2007, 98, 266802.

34 Nordlander, P.; Oubre, C.; Prodan, E.; Li, K.; Stockman, M. I. Plasmon Hybridization in Nanoparticle Dimers. Nano Lett. 2004, 4, 5, 899-903.

35 Ye, J.; Van Dorpe, P.; Lagae, L.; Maes, G.; Borghs, G. Observation of Plasmonic Dipolar Anti-Bonding Mode in Silver Nanoring Structures. Nanotech. 2009, 20, 465203.

36 Park, T.-H.; Nordlander, P. On the Nature of the Bonding and Antibonding Metallic Film and Nanoshell Plasmons. Chem. Phys. Lett. 2009, 472, 228-231.

37 Zhang, S.; Genov, D. A.; Wang, Y.; Liu, M.; Zhang, X. Plasmon-Induced Transparency in Metamaterials. Phys. Rev. Lett. 2008, 101, 047401.

38 Liu, N.; Langguth, L.; Weiss, T.; Kästel, J.; Fleischhauer, M.; Pfau, T.; Giessen, H. Plasmonic Analogue of Electromagnetically Induced Transparency at the Drude Damping Limit. Nat. Mater. 2009, 8, 758-762.

39 Abb, M.; Albella, P.; Aizpurua, J.; Muskens, O. L. All-Optical Control of a Single Plasmonic Nanoantenna-ITO Hybrid. Nano Lett. 2011, 11, 2457-2463.

40 Juvé, V.; Scardamaglia, M.; Maioli, P.; Crut, A.; Merabia, S.; Joly, L.; Del Fatti, N.; Vallée, F., Cooling Dynamics and Thermal Interface Resistance of Glass-Embedded Metal Nanoparticles. Phys. Rev. B 2009, 80, 195406.

41 Lassiter, J. B.; Sobhani, H.; Fan J. A.; Kundu J.; Capasso F.; Nordlander P.; Halas N. J. Fano Resonances in Plasmonic Nanoclusters: Geometrical and Chemical Tunability. Nano Lett. 2010, 10, 3184-3189.

42 Garcia de Abajo, F. J.; Howie, A. Relativistic Electron Energy Loss and Electron-Induced Photon Emission in Inhomogeneous Dielectrics. Phys. Rev. Lett. 1998, 80, 5180-5183.

43 Arbouet, A.; Christofilos, D.; Del Fatti, N.; Vallé, F.; Huntzinger, J. R.; Arnaud, L.; Billaud, P.; Broyer, M. Direct Measurement of the Single-Metal-Cluster Optical Absorption. Phys. Rev. Lett. 2004, 93, 127401. 
44 Muskens, O. L.; Del Fatti, N.; Vallé, F.; Huntzinger, J. R.; Broyer, M. Single Metal Nanoparticle Absorption Spectroscopy and Optical Characterization. Appl. Phys. Lett. 2006, 88, 063109.

\section{Figures}



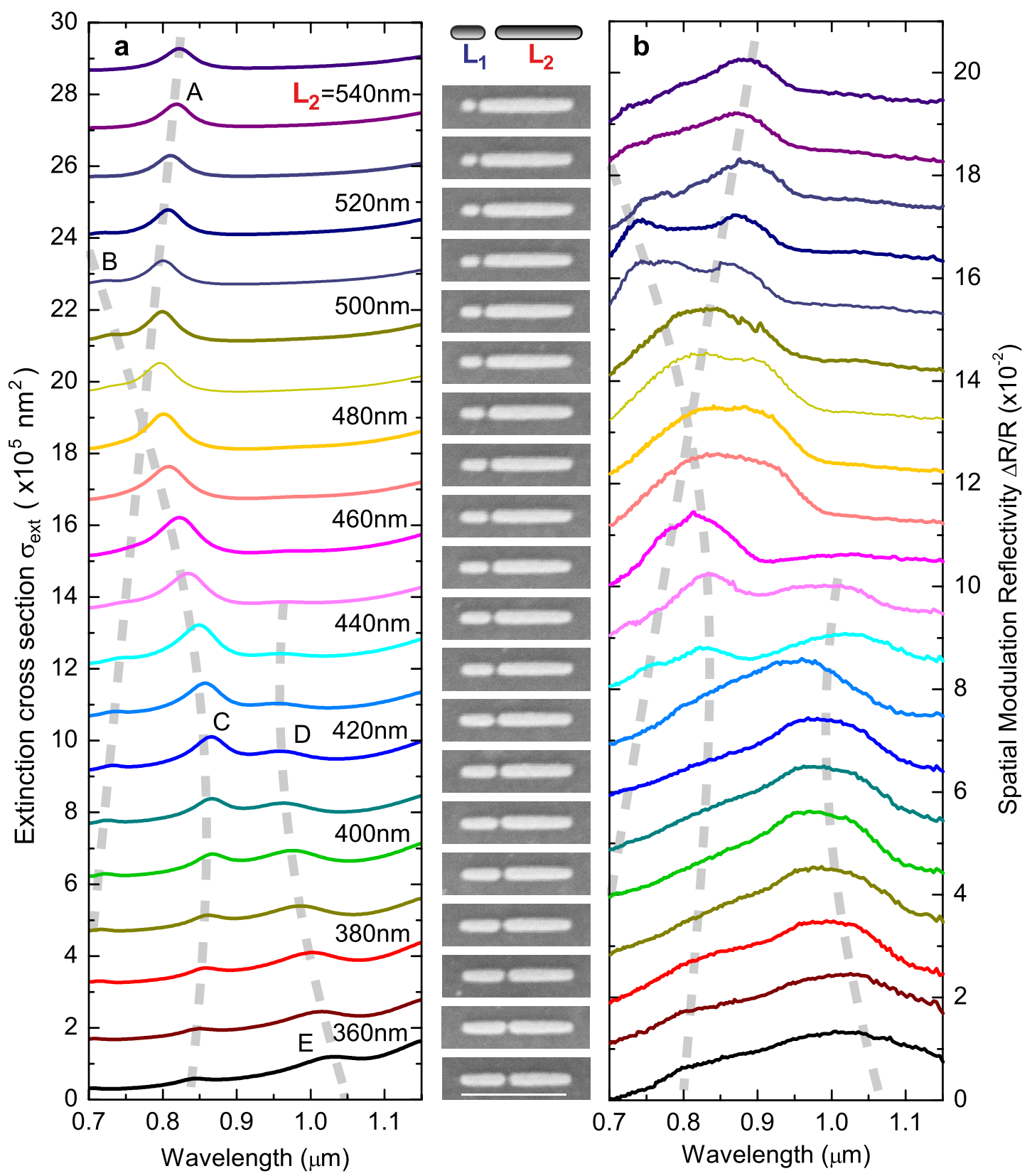

FIG. 1: Theoretical (a) and experimental (b) extinction spectra of individual asymmetric antennas composed of metallic rods of different length $L_{1}$ (short rod) and $L_{2}$ (long rod). Corresponding SEM images are shown in the center. The antennas are separated by a $20 \mathrm{~nm}$ gap, i. e. they are in an efficient coupling regime. The total length of both antenna arms is kept constant at $L_{t o t}=600 \mathrm{~nm}$ excluding the gap. The length of the long rod $L_{2}$ varies from $360 \mathrm{~nm}$ (bottom) to $540 \mathrm{~nm}$ (top), with the length of the short rod $L_{1}$ decreasing accordingly, as observed in the SEM pictures. A set of surface modes labeled from A to E are identified in the theoretical spectra. Dashed lines are guides of the modes to the eye. 

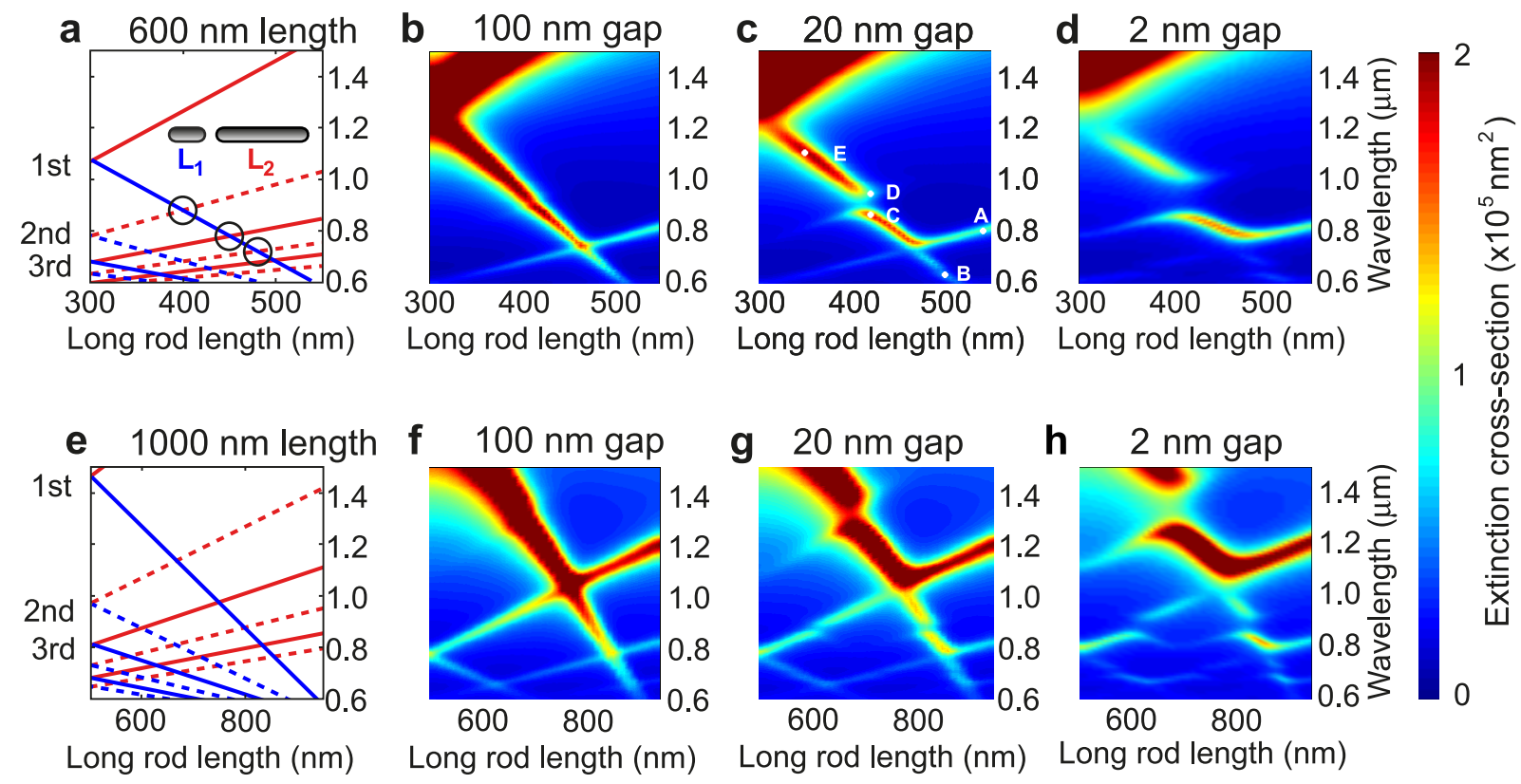

FIG. 2: (a-c) Spectral position of the resonances for an asymmetric dimer of total length $L_{\text {tot }}=$ $600 \mathrm{~nm}$ as estimated from the scaling law by Novotny ${ }^{33}$ applied to isolated antennas (a), as well as from full electrodynamical calculations of asymmetric antennas weakly interacting (b), and strongly interacting (c,d). (e-h) Same as (a-d) for a total length of $L_{\text {tot }}=1000 \mathrm{~nm}$. The modes of the smaller rod are shown in blue in (a) and (e) and the modes of the longer rod in red. Bright modes are displayed in full lines, whereas dark modes are displayed in dashed lines. 


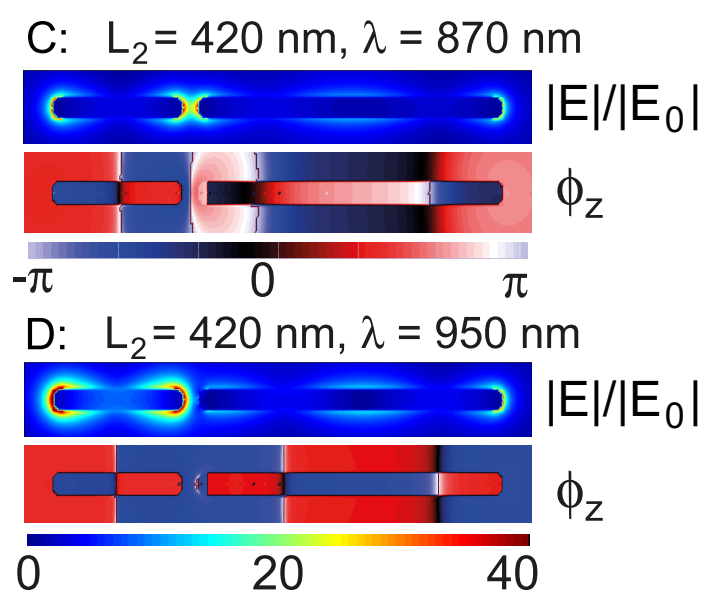

FIG. 3: Near-field enhancement modulus and phase distribution in the plane perpendicular to the incoming wave at the spectral position of the splitting modes (modes $\mathrm{C}$ and D) for $L_{\text {tot }}=600 \mathrm{~nm}$ and $20 \mathrm{~nm}$ gap. 

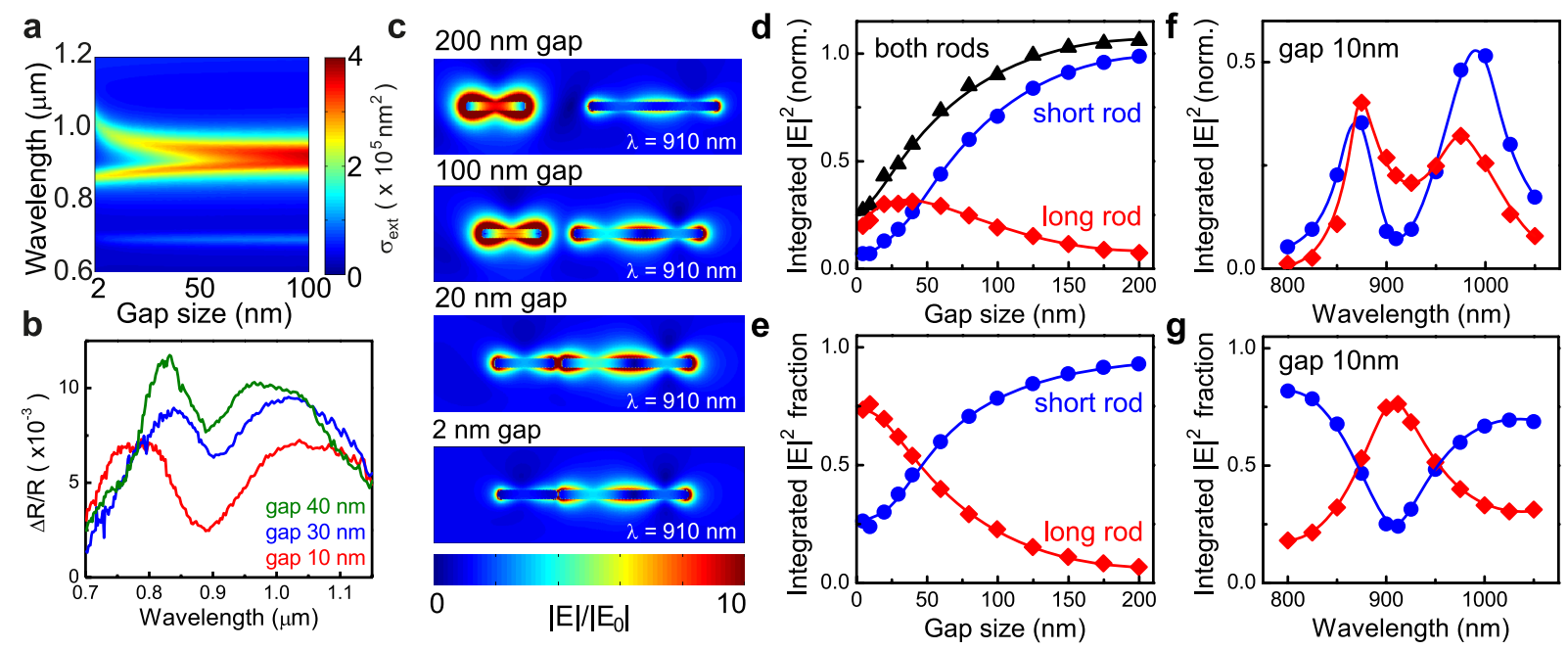

FIG. 4: a) Extinction cross-section of an asymmetric antenna with $L_{2}=410 \mathrm{~nm}$ and $L_{\text {tot }}=600 \mathrm{~nm}$ as a function of gap size. b) Experimental spectra of differential reflectance for the same asymmetric antenna for three different gap sizes. (c) Near-field intensity maps at a wavelength of $910 \mathrm{~nm}$, i.e. the center of the EIT window, were calculated for the same length ratio as in a), with gaps from 200 $\mathrm{nm}$ (top) down to $2 \mathrm{~nm}$ (bottom). Even at large gaps such as $200 \mathrm{~nm}$, there is still an excitation of the second-order mode of the long nanorod. For very small gap sizes, we observe a suppression of the near-field enhancement in the gap and a stronger excitation of the near-field around the longer rod. (d,e) Integrated local-field intensity $|E|^{2}$ at 2-nm distance around the short and long rods in the asymmetric dimers of (c) as a function of gap size. (d) is normalized to the total integrated $|E|^{2}$ of the two rods for infinite gap, (e) is the same data but normalized to the total integrated $|E|^{2}$ of the two rods at each gap size. Energy transfer from the bright $\lambda_{\text {eff }} / 2$ mode of the short rod to the dark $3 \lambda_{\text {eff }} / 2$ mode of the long rod is observed with up to $76 \%$ efficiency. (f,g) Same as (d,e) as a function of wavelength and for a fixed gap size of $10 \mathrm{~nm}$. 

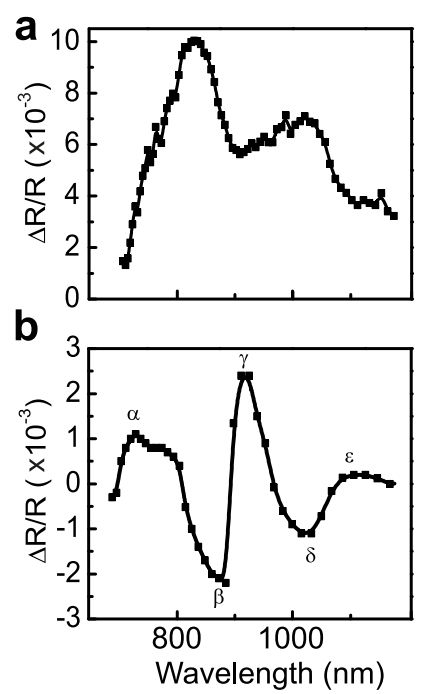

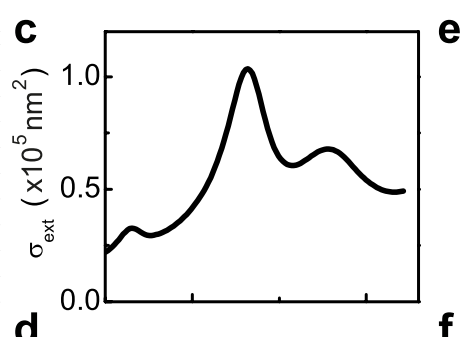

d

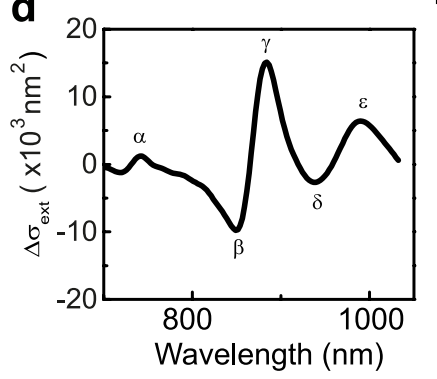

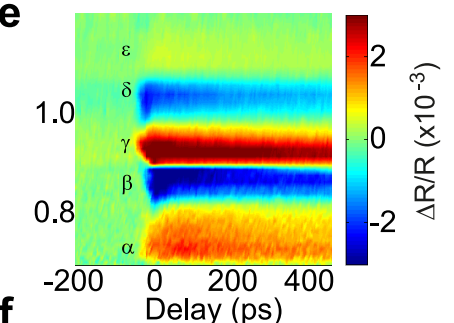

f

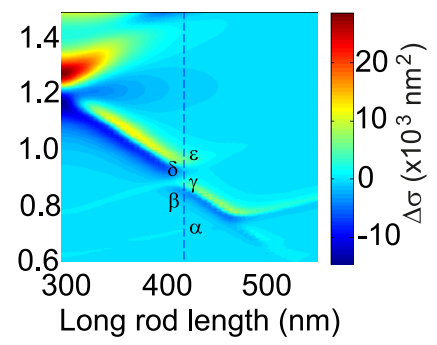

FIG. 5: a) Experimental spectra of differential reflectance $\Delta R / R$ acquired by spatial modulation spectroscopy for a dimer with interference between the first order mode of the shorter rod segment and the second order mode of the longer rod segment. b) Corresponding nonlinear signal for pumping energy of $60 \mathrm{pJ}$ probed with a second pulse at 5 ps delay after the pump pulse arrives, showing the complex dynamics taking place. Symbols $\alpha-\epsilon$ mark the spectral maxima and minima in the nonlinear response for comparison between experiment and theory. c) and d) show the corresponding spectra obtained with FDTD simulations. In d), the spectrum represented is the differential extinction cross section for a variation of $\Delta N=-6 \times 10^{19} \mathrm{~cm}^{-3}$ in free carrier density in the ITO. e) Experimental time resolved trace of the nonlinear signal. f) Difference in extinction cross-section for an asymmetric dimer of varying lengths using BEM simulations for the same change of free carrier concentrations in the ITO. 


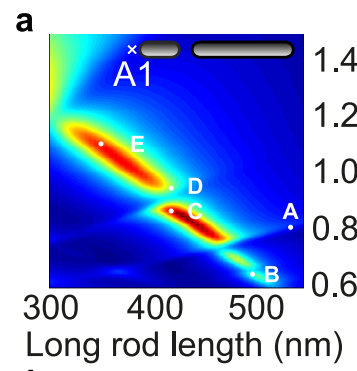

d

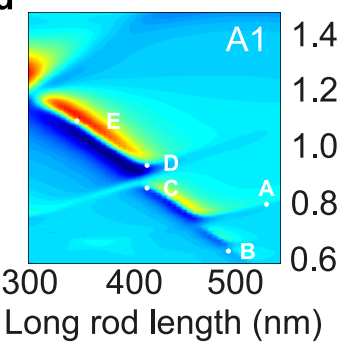

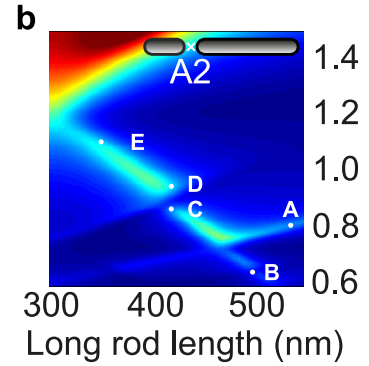

e
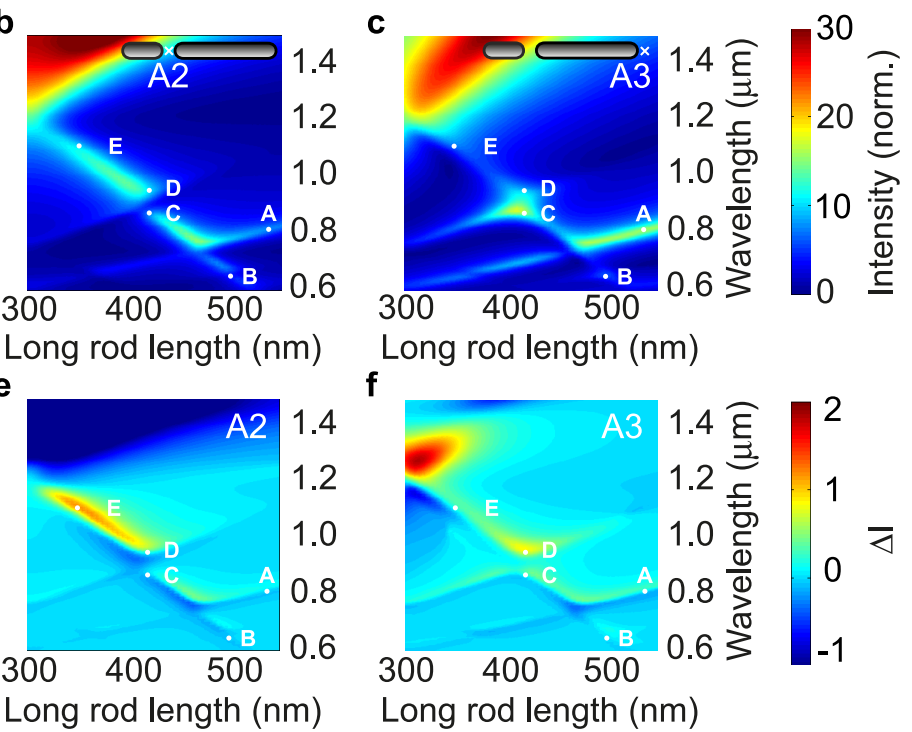

FIG. 6: (a-c) Calculated near-field intensity maps, for a coupled dimer antenna of $L_{\text {tot }}=600 \mathrm{~nm}$ and gap width of $20 \mathrm{~nm}$, as a function of the long arm length $L_{2}$. Maps were taken at three positions respectively at a distance of $5 \mathrm{~nm}$ from the short tip end (A1), in the center of the gap (A2) and at $5 \mathrm{~nm}$ from the long rod of the dimer (A3). (d-f) Nonlinear change in near-field intensity $\Delta I=I-I_{0}$ as calculated in the points A1-A3. For the calculations of $I$ and $I_{0}$ we used values for the free-electron density of ITO of $N=6.7 \times 10^{20} \mathrm{~cm}^{-3}$ and $N_{0}=7.3 \times 10^{20} \mathrm{~cm}^{-3}$, respectively. 


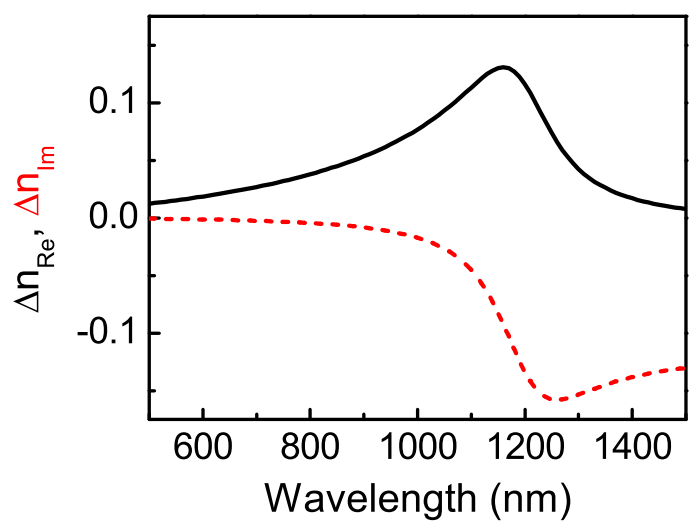

FIG. 7: Real part (black line) and imaginary part (red dashed line) of the nonlinear refractive index change of ITO used to model the nonlinear pump-probe spectra of Fig. 5 and Fig. 6. Values are obtained using the Drude-model by subtracting refractive index values at free-electron density of ITO of $N=6.7 \times 10^{20} \mathrm{~cm}^{-3}$ and $N_{0}=7.3 \times 10^{20} \mathrm{~cm}^{-3}$. 

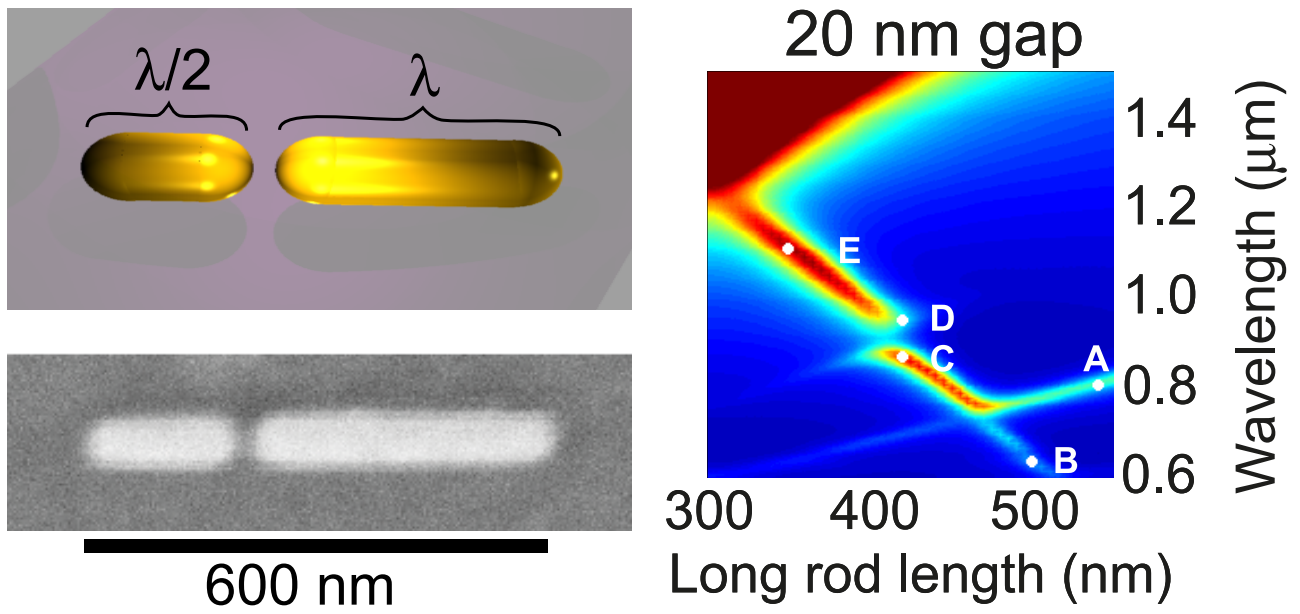

FIG. 8: Table of contents figure. 